\title{
AN ILL-POSED CAUCHY TYPE PROBLEM FOR AN ELASTIC STRIP
}

\author{
A.N. Galybin ${ }^{1}$ and G.A Rogerson ${ }^{2}$ \\ ${ }^{1}$ The Schmidt Institute of Physics of the Earth, B.Gruzinskaya 10-1, Moscow, 123242, Russia \\ ${ }^{2}$ School of Computing and Mathematics, Keele University, Keele, Staffordshire, ST5 5BG, UK
}

\begin{abstract}
This study deals with an incorrectly posed, plane elasticity, boundary value problem for a strip. The strip is loaded by a concentrated load of known intensity applied to one side and the displacements on this side are also known. The problem is therefore over-determined on one side of the boundary; in contrast no boundary conditions are specified on the other side of the strip. Therefore, the problem is ill-posed with the specified boundary conditions.

The problem can be reduced to a system of integral equations derived from basic properties of holomorphic functions, which are used to prove uniqueness of the considered boundary value problem.

An analytical solution of the problem is obtained by applying Fourier transforms. The inversion of the Fourier transform is performed with the use of the Stieltjes integral. This is a non-stable operation, which necessitates the application of a regularisation technique in order to build stable solutions. For numerical implementation we discuss the regularisation procedure based on the SVD truncation method.
\end{abstract}

Keywords: crack detection; elastostatics; ill-posed problems; integral equations; integral transforms;

\section{Introduction}

Crack detection on the interfaces between dissimilar materials is one of the classic problems in the mechanics of coated bodies. A number of methods, based on different physical phenomena, can be deployed for such problems, among them x-ray, electric and acoustic methods, analysis of frequency response functions and other non-destructive techniques. This paper is devoted to the development of an elastostatic approach, employing displacement monitoring on the stress-free surfaces of an object. A consequence of this is that the boundary value problem, BVP, for an elastic body becomes over-determined on the stress-free part of the boundary. In contrast, it may be underdetermined on the remaining part of the boundary, where the appearance of cracks, due to the uncertainty in the boundary conditions that can be posed there, can be expected. In general, no boundary conditions are required on this part of the boundary as the displacement monitoring of the stress-free boundary provides sufficient information to determine the stress-strain state of the elastic body. We remark that it has been shown by Shvab (1989) that this problem can be reduced to a Cauchy problem for the Laplace operator (see also his other paper (Schwab, 1994), however note a different spelling of the authors name). The latter problem belongs to a wide class of conditionally well-posed BVP's as its solution in not stable with respect to small perturbations in boundary conditions.

In accordance with Muskhelishvili (1963) there are three main classical types of plane elastic BVP: 
- $1^{\text {st }}$ fundamental problem: stress vector (or tractions) is given on the entire boundary of the domain;

- $\quad 2^{\text {nd }}$ fundamental problem: displacements (or their contour derivatives) are given on the entire boundary;

- $\quad 3^{\text {rd }}$ (mixed) problem: the stress vector is given on part of the boundary and the displacements on the remaining part.

Other formulations are often found in contact problems, e.g. Johnson (1985). They assume combinations of stress and displacement components, e.g. normal stress and tangential displacement, or normal displacements and shear stresses. Winkler's type of boundary conditions assumes linear relationships between the normal and shear stress components and the displacement components.

In all classical formulations two boundary conditions should be specified on the entire boundary of the plane domain. All these problems are well posed, which means that they possess unique and stable solutions.

Classical problems are not the only BVP that can be considered for a plane elastic domain. There exists quite a wide class of ill-posed problems. For instance, a BVP with incomplete boundary conditions that can possess non-unique solutions, see e.g. Galybin \& Mukhamediev (1999) or Galybin (2011). BVP's with unstable solutions are common in all applications where boundary data are not fully available on the whole boundary of a body (including internal boundaries). This means that the number of scalar boundary conditions is different from the problem dimension, i.e. for plane domain three or four conditions on part of the boundary and respectively one or none on the rest of the boundary. In solid mechanics, such formulations can deal with identification of elastic moduli, model parameters or buried objects, for instance, cracks, inclusions or other defects. One can also mention contributions by Tanaka \& Masuda (1986) for identification of flaw shape in a body, Gao \& Mura (1991) for analysing plastic flow near crack tips, Hsieh \& Mura (1993) for nondestructive cavity identification, Tsvelodub (2000) for identification of a non-linear inclusion; also see Kubo (1988) for an overview of inverse problems in fracture mechanics, and Bonnet \& Constantinescu (2005) for a general overview of engineering problems leading to ill-posed formulations.

Methods based on integral equations are often used for solving the above mentioned types of BVP's, see, for instance, Zabaras et al (1989) and Galybin (2016) for contact problems, Galybin (1999) for a wedge-like elastic domains, Yeih et al (1993) and Bui (1994) for providing Fredholm integral equations. Finite element formulations are also common, see references (Maniatty et al, 1989; Maniatty \& Zabaras, 1989; Schnur \& Zabaras, 1990) for details. Regardless of the method of solution, the problem remains ill-posed because of high sensitivity of the solution to small variations of boundary data, which requires the development of different approaches capable of obtaining stable solutions, see Tikhonov \& Arsenin (1977) for more detail. Such approaches can employ regularisation techniques, iterative methods and algorithms, such as those discussed in references (Marin et al, 2001; Marin \& Lesnic, 2002; Marin \& Lesnic, 2005; Galybin, 2001). In particular, high efficiency of the SDV truncation method has been confirmed.

This study is aimed at the development of a numerical technique for identification of interface cracks in coated bodies and focused on solving an ill-posed problem for an elastic strip. We present a semi-analytical approach for the case when the strip is loaded by a concentrated load and the displacements are monitored on the stress-free side, while no boundary conditions are specified on another side. An example of obtaining a stable solution by the SVD regularisation is provided. 


\section{Formulation}

\subsection{Problem description}

We consider an elastic base coated by a relatively thin film made of a different elastic material. In many cases when the curvature of the base is not an important factor, it is sufficient to approximate the base by elastic half-plane. The film can then be viewed as a strip of the thickness $2 h$. It is assumed that the strip is loaded by a concentrated load $P$ directed normal to the surface, as shown in Fig.1.

Let the strip be fully bonded to the half-plane. Then, in mechanical terms, this indicates continuity of the stress and displacement vectors across the interface under any load applied on the strip surface. As a consequence, the stress distribution along the interface is continuous, as shown in Fig 1(a). In the case of partial delamination of the interface, the stress distribution becomes discontinuous. For the case of a crack-like delamination the stresses are unbounded at the crack tips, as shown in Fig 1(b). Note that the stress profiles are qualitative, they vary depending on load applied, thickness and elastic moduli. In any case the stress profiles should provide equilibrium of the strip, in particular the integral of the normal stresses over the interface is equal to the applied load $P$.

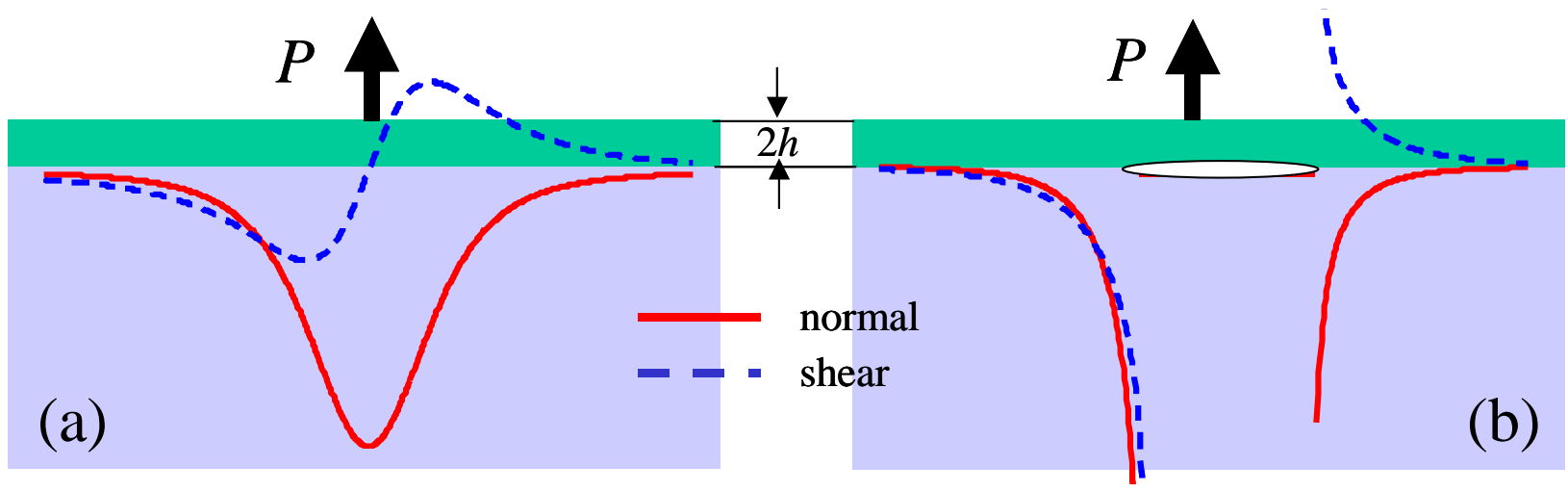

Figure 1 Qualitative profiles of normal stresses (solid) and shear stresses (dashed) on the interface: (a) for full bounding, (b) for delamination (crack) on the interface.

In the case of a fully bonded strip and interface, the solution of the direct problem depicted in Fig 1(a) can be obtained in analytical form by applying the Fourier transform with respect to the coordinate along the interface. In particular, it is found in Shevljakov (1977) as a special case of multi-layered elastic media. Asymptotic solutions are available in Kaplunov et al (2018), where the references to other papers can be found. Solutions for the case depicted in Fig 1(b) can only be obtained numerically; examples of the calculations of the stress intensity factors are found in Erdogan \& Gupta (1971), also see handbook (Murakami, 1987) and references therein.

Apart from a special case of loading reported by Khrapkov (2001), none of the references above supply data on the displacement distribution along the upper surface of the strip; this is the information we need to formulate the ill-posed problem formulated below. The direct solutions are out of the scope of this paper since we intend to use data on displacement monitoring on the upper side of the strip as the additional boundary conditions that will enable us to calculate the stress-state of the strip. However, the solution of the $1^{\text {st }}$ BVP is presented in Appendix B, providing the possibility to use it for the generation of synthetic data on displacement monitoring in future research.

The Kolosov-Muskhelishvili formulas for the stress and displacement components in a Cartesian coordinate system $O x y$ are as follows (Muskhelishvili, 1963): 


$$
\begin{aligned}
& \frac{\sigma_{x}+\sigma_{y}}{2}=\Phi(z)+\overline{\Phi(z)}, \quad \frac{\sigma_{y}-\sigma_{x}}{2}+i \tau_{x y}=\bar{z} \Phi^{\prime}(z)+\Psi(z), \\
& 2 G(u+i v)=\kappa \varphi(z)-z \overline{\Phi(z)}-\overline{\psi(z)}, \quad \Phi(z)=\varphi^{\prime}(z), \quad \Psi(z)=\psi^{\prime}(z) .
\end{aligned}
$$

Here $\sigma_{x}, \sigma_{y}, \tau_{x y}$ are the stress components, and $u$ and $v$ are displacement components along the $x$ and $y$ axes respectively, $G$ is shear modulus, $\kappa$ is equal to (3-4v) for the plain stress and to (3$v) /(1+v)$ for the plane stress conditions, $v$ is Poisson's ratio.

Let $t$ be a point on a contour. Then the stress vector, $N+i T$, and the contour derivative of the displacement vector, $u^{\prime}+i v^{\prime}$, on the contour have the form (e.g., Muskhelishvili, 1963):

$$
\begin{aligned}
& N(t)+i T(t)=\Phi(t)+\overline{\Phi(t)}+e^{-2 i \theta(t)} \overline{D(t)}, \\
& 2 G\left[u^{\prime}(t)+i v^{\prime}(t)\right]=\kappa \Phi(t)-\overline{\Phi(t)}-e^{-2 i \theta(t)} \overline{D(t)} .
\end{aligned}
$$

The angle $\theta$ is the angle between the tangent to the contour passing through the point $t$ and the positive direction of the $x$-axis of an arbitrary situated Cartesian coordinate system $O x y$. This angle is counted anti-clockwise, and can be express through the contour derivatives as follows:

$$
\frac{d t}{d \bar{t}}=e^{2 i \theta(t)}
$$

The function $D(t)$ is the boundary value of the complex stress deviator function

$$
D(z, \bar{z})=(\bar{z}-z) \Phi^{\prime}(z)+\Omega(z),
$$

where for the sake of convenience we introduced another holomorphic function

$$
\Omega(z)=z \Phi^{\prime}(z)+\Psi(z) .
$$

By summing the equations in eqn (2) one finds:

$$
(1+\kappa) \Phi(t)=N(t)+i T(t)+2 G\left[u^{\prime}(t)+i v^{\prime}(t)\right]
$$

Therefore the boundary value of the complex potential is fully defined by the displacement and the stress vector. A somewhat similar expression can be derived for the internal points:

$$
(\kappa+1) \Phi(z)=2 G\left(u_{x}^{\prime}+i v_{x}^{\prime}\right)+\sigma_{y}-i \tau_{x y}
$$

where $u_{x}^{\prime}+i v_{x}^{\prime}$ are derivatives of the displacement vector in the direction of the real axis.

\subsection{Auxiliary problem for holomorphic functions}

Let us consider a smooth closed contour $\Gamma$ that separates the entire complex plane into interior $\mathrm{X}^{+}$ and exterior $\mathrm{X}^{-}$regions. Let a complex-valued function $\varphi(t)$ satisfying the Holder condition be given on $\Gamma$. It is known (e.g., Gakhov, 1990) that the function $\varphi(t)$ is the boundary value of holomorphic functions $\varphi^{ \pm}(z)$ in $\mathrm{X}^{ \pm}$if and only if:

$$
\varphi(\zeta)= \pm \frac{1}{\pi i} \int_{\Gamma} \frac{\varphi(t)}{t-\zeta} d t, \quad \zeta \in \Gamma, \quad \varphi(\infty)=0 .
$$

Here and further the signs " \pm " are chosen according to $\varphi^{ \pm}(z)$, i.e. $z \in X^{ \pm}$.

If $\varphi(\infty) \neq 0$ then (8) remains valid for $\mathrm{X}^{+}$but should be corrected for $\mathrm{X}^{-}$by adding $\varphi(\infty) / 2$ to the left hand side. It is also valid if the contour $\Gamma$ is an arbitrary smooth curve going to infinity, e.g. the real (or imaginary) axis. 
We further separate the contour into two non-intersecting parts, $\Gamma_{1}$ and $\Gamma_{2}$, such that $\Gamma=\Gamma_{1} \cup \Gamma_{2}$ and consider the following problem. Find a function $\varphi^{+}(z)\left(\right.$ or $\left.\varphi^{-}(z)\right)$ which is holomorphic inside (or outside) the domain $X^{+}$(or $\left.X^{-}\right)$by its boundary value $\varphi^{+}(t)=\varphi_{1}(t)\left(\right.$ or $\left.\varphi^{-}(t)=\varphi_{1}(t)\right)$ given on a part of the domain boundary $\Gamma_{1}$ (while nothing is given on the other part $\Gamma_{2}$ ).

The solution of this problem can be readily obtained by analytical continuation if one has an exact analytical expression for the function $\varphi^{+}(\mathrm{z})\left(\operatorname{or}^{-}(\mathrm{z})\right)$ ). However if no analytical expression for them is known and its values are only given on a set of discrete points, then the solution would depend on the way the discrete values were converted into an analytic expression. The question regarding stability of the solution also arises, Tikhonov \& Arsenin (1977). Even if the exact solution is stable, the method of analytic continuation of the boundary values can affect stability of the solution. Further, we try to find an appropriate approximation for the case of an elastic strip. However, let us first briefly discuss the method of analytic continuation for general domains. It is based on consideration of the integral equations that follow from the basic properties of holomorphic functions.

The integral on the right-hand side of eqn (8) can be decomposed into the sum of two integrals over the parts $\Gamma_{1}$ and $\Gamma_{2}$ of the whole boundary $\Gamma$. On $\Gamma_{2}$, values of the functions $\varphi^{ \pm}(t)$ are unknown and can be denoted by $\varphi_{2}(t)$. Then the following system of integral equations can be obtained from eqn (8) for determination of the unknown function $\varphi_{2}(t)$ :

$$
\left\{\begin{array}{l}
\frac{1}{\pi i} \int_{\Gamma_{2}} \frac{\varphi_{2}(t)}{t-\zeta} d t= \pm \varphi_{1}(\zeta)-\frac{1}{\pi i} \int_{\Gamma_{1}} \frac{\varphi_{1}(t)}{t-\zeta} d t, \quad \zeta \in \Gamma_{1}, \\
\varphi_{2}(\zeta) \mp \frac{1}{\pi i} \int_{\Gamma_{2}} \frac{\varphi_{2}(t)}{t-\zeta} d t= \pm \frac{1}{\pi i} \int_{\Gamma_{1}} \frac{\varphi_{1}(t)}{t-\zeta} d t, \quad \zeta \in \Gamma_{2} .
\end{array}\right.
$$

The equations of the system are of different type. The first can be referred to as from the class of Fredholm's equations, with a kernel that has fixed singularities at the end points. The second is a dominant singular integral equation with the Cauchy type kernel on an open contour. Despite this fact they are equivalent, which can be readily proved as follows. Let $\varphi_{2}{ }^{\prime}(t)$ and $\varphi_{2}{ }^{\prime \prime}(t)$ be two different solutions of the first and second equation, respectively. Then there exist two different holomorphic functions $\varphi^{\prime}(\mathrm{z})$ and $\varphi^{\prime \prime}(\mathrm{z})$ that have different boundary values on $\Gamma_{2}$. Therefore, their difference $\varphi_{0}(\mathrm{z})=\varphi^{\prime}(\mathrm{z})-\varphi^{\prime \prime}(\mathrm{z})$ is also holomorphic in $\mathrm{X}^{+}$(or $\mathrm{X}^{-}$depending on the sign in (9)). Bearing in mind that $\varphi_{0}(\mathrm{t})=0$ on $\Gamma_{1}$, one can conclude, in accordance with Muskhelishvili (1963), that $\varphi_{0}(z)=0$ everywhere in the domain. This confirms that the assumption $\varphi_{2}{ }^{\prime}(t) \neq \varphi_{2}{ }^{\prime \prime}(t)$ is not valid. Hence $\varphi_{2}{ }^{\prime}(t)=\varphi_{2}{ }^{\prime \prime}(t)$ and that completes the proof. Thus, the first equation can be considered as an alternative regularisation of the dominant singular integral equation (presented by the second equation in (9)) performed on a different contour, which is different from the conventional regularisation.

\subsection{Problem formulation for an elastic strip}

Let us consider an elastic strip $\mathrm{X}=\{z=x+i y:-\infty<x<\infty, 0 \leq y \leq 2 h\}$ with the boundaries $\Gamma_{1}=\{z=t:-\infty<t<\infty\}, \quad \Gamma_{2}=\{z=t+2 i h:-\infty<t<\infty\}$. The strip is loaded as shown in Fig. 2, however the stresses on $\Gamma_{2}$ are unknown. Instead it is assumed that the displacements on the lower boundary are known. Therefore no conditions are prescribed on $\Gamma_{2}$ and the following boundary conditions take place on $\Gamma_{1}$ :

$$
\sigma_{y}(x)=P \delta(x), \quad \tau_{x y}(x)=0, \quad 2 G\left[u^{\prime}(x)+i v^{\prime}(x)\right]=w(x),
$$

where $P$ is the applied (known) load, $\delta(x)$ is the Dirac delta function and $w=w(x)$ is a known 
complex valued function. This complex valued function could be subject to errors as the result of measurements and differentiation.

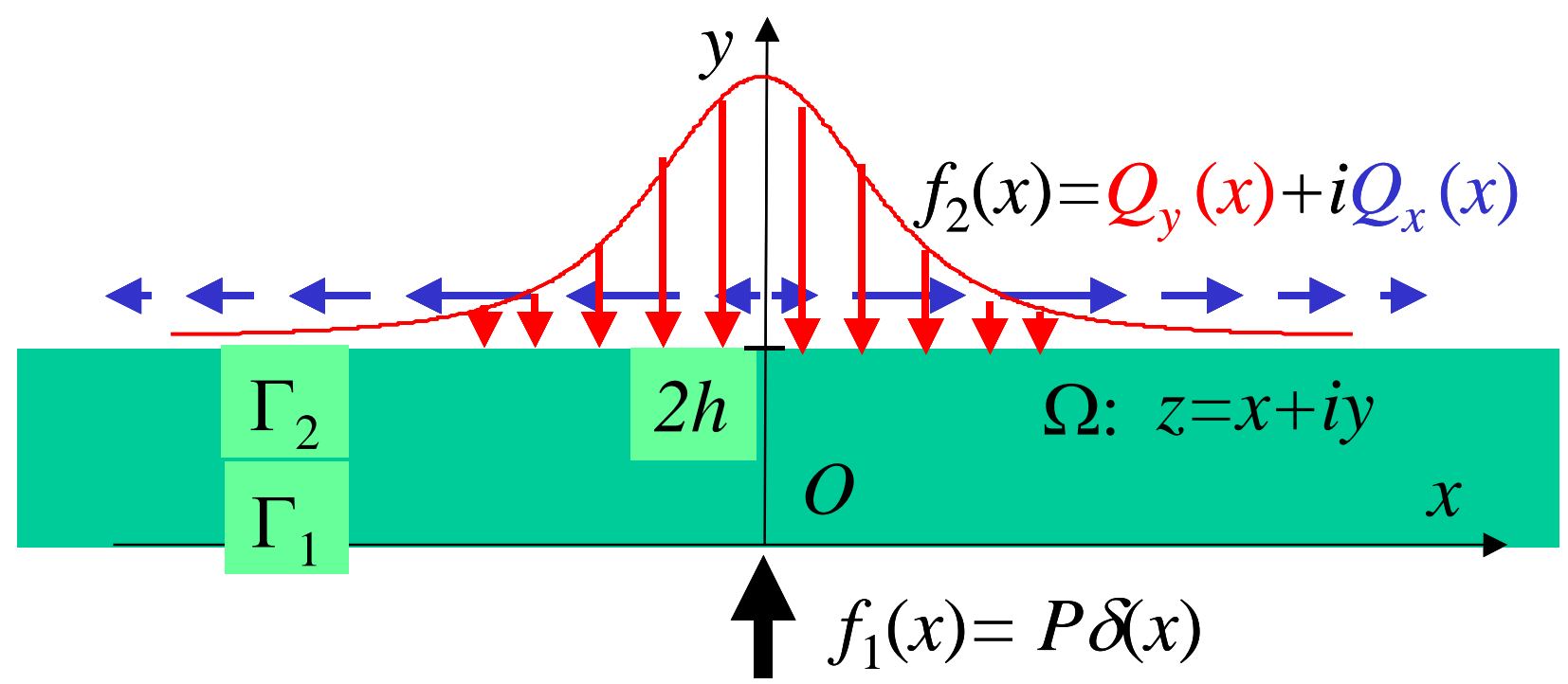

Figure 2 Strip of the thickness $2 h$ under concentrated load $P$ on the lower boundary $\Gamma_{1}$ and unknown loads $Q_{y}(x), Q_{x}(x)$ on the upper boundary $\Gamma_{2}$.

It should be noted that the conditions in (10) allow for the determination of the tangential stress $\sigma_{x}$ on $\Gamma_{1}$, which is as follows:

$$
\sigma_{x}(x)=\frac{4}{\kappa+1} \operatorname{Re} w(x)+\frac{3-\kappa}{1+\kappa} P \delta(x) .
$$

Therefore, all components of stresses, strain and displacements are known on the lower boundary, which indicates a Cauchy-type initial value problem.

The above problem can be further reduced to the problem for the determination of the holomorphic function $\Phi=\Phi(\mathrm{z})$ by its known boundary value on $\Gamma_{1}$ (see previous subsection):

$$
\Phi(x)=(1+\kappa)^{-1}[P \delta(x)-w(x)], \quad-\infty<x<\infty .
$$

As soon as $\Phi(\mathrm{z})$ is found, it is then possible to formulate a similar problem for the determination of the holomorphic function $\Omega(z)$. Indeed, it follows from the second formula in (1), (4) and (11) that on the lower boundary:

$$
\Omega(x)=\frac{\sigma_{y}-\sigma_{x}}{2}=\frac{\kappa-1}{\kappa+1} P \delta(x)-\frac{2}{\kappa+1} \operatorname{Re} w(x) .
$$

As long as both potentials are found this completes the solution.

\section{Solution for the strip}

\subsection{Fourier transform}

The Fourier transform, $\mathbf{F}($.$) , and its inverse, \mathbf{F}^{-1}($.$) , are specified as follows (Bateman \& Erdelyi,$ 1954):

$$
\mathbf{F}(Y(x)) \equiv \hat{Y}(s)=\int_{-\infty}^{\infty} Y(x) e^{-i s x} d x, \quad \mathbf{F}^{-1}(\hat{Y}(s)) \equiv Y(x)=\frac{1}{2 \pi} \int_{-\infty}^{\infty} \hat{Y}(s) e^{i s x} d s
$$


where $s$ is a real parameter $(\operatorname{Im}(s)=0)$.

The following property of (14) should be mentioned:

$$
F(\overline{Y(x)})=\overline{Y(s)}=\int_{-\infty}^{\infty} \overline{Y(x)} e^{-i s x} d x=\overline{\int_{-\infty}^{\infty} Y(s) e^{i s x} d s}=\overline{\hat{Y}(-s)}
$$

In particular, if $Y_{0}(x)$ is real valued then

$$
\overline{\hat{Y}_{0}(s)}=\hat{Y}_{0}(-s) \text {. }
$$

The Fourier transform (FT) of any holomorphic function $\omega(\mathrm{z})=\omega(x+i y)=\omega(x, y)$, with respect to the variable $x$, can formally be presented in the form:

$$
\mathbf{F}(\omega(z))=\int_{-\infty}^{\infty} \omega(x+i y) e^{-i s x} d x=e^{-s y} \int_{-\infty}^{\infty} \omega(x+i y) e^{-i s(x+i y)} d x=e^{-s y} \hat{\omega}(s, 0) .
$$

The rigorous proof follows by applying FT to the Cauchy-Riemann conditions. Indeed, it can be shown that the derivative of any holomorphic function with respect to the complex conjugated variable vanishes, $\partial_{\bar{z}} \omega(z)=0$, implying that

$$
\mathbf{F}\left(\partial_{x} \omega(x+i y)+i \partial_{y} \omega(x+i y)\right)=0 \Rightarrow i s \hat{\omega}(s, y)+i \hat{\omega}_{y}^{\prime}(s, y)=0 \Rightarrow \hat{\omega}(s, y)=\hat{\omega}(s, 0) e^{-s y} .
$$

In view of the FT properties one notes the following:

$$
\mathbf{F}(\Phi(z))=e^{-s y} \hat{\Phi}(s, 0), \quad \mathbf{F}\left((\bar{z}-z) \Phi^{\prime}(z)\right)=2 y s e^{-s y} \hat{\Phi}(s, 0), \quad \mathbf{F}(\Omega(z))=e^{-s y} \hat{\Omega}(s, 0) .
$$

Taking into account that $\mathbf{F}(\delta(x))=1$ (hence $\mathbf{F}^{-1}(1)=\delta(x)$ ) one arrives at the following two conditions:

$$
\hat{\Phi}(s, 0)=\frac{P+\hat{w}(s)}{\kappa+1}, \quad \hat{\Omega}(s, 0)=\frac{\kappa-1}{\kappa+1} P-\frac{\hat{w}(s)+\overline{\hat{w}(-s)}}{\kappa+1} .
$$

On the boundary $\Gamma_{2}$

$$
\hat{\Phi}(s, y+2 i h)=e^{-2 h y} \frac{P+\hat{w}(s)}{\kappa+1}, \quad \hat{\Omega}(s, y+2 i h)=e^{-2 h y} \frac{\kappa-1}{\kappa+1} P-e^{-2 h y} \frac{\hat{w}(s)+\overline{\hat{w}(-s)}}{\kappa+1} .
$$

It is worth noting that these equations can readily be derived from the general system (9), see Appendix A.

By applying the inverse FT to (21) one finds the following formal solutions for the holomorphic functions everywhere in the strip, including its boundaries:

$$
\Phi(z)=\frac{P}{(1+\kappa) z}+\frac{1}{(1+\kappa) 2 \pi} \int_{-\infty}^{\infty} \hat{w}(s) e^{i s z} d s, \quad \Omega(z)=\frac{\kappa-1}{\kappa+1} \frac{P}{z}-\frac{1}{(1+\kappa) 2 \pi} \int_{-\infty}^{\infty}(\hat{w}(s)+\overline{\hat{w}(-s)}) e^{i s z} d s .
$$

It is evident from (22) that the potentials can be found by analytical continuation, i.e. by replacing the variable $x$ in the boundary conditions by the complex variable $z$. However, this can only be done if the function $w(x)$ is given in analytical form. Otherwise one needs to calculate the inverse FT of the function $\hat{w}(s) e^{-s y}(0 \leq y \leq 2 h)$. In order to exist, the function $\hat{w}(s)$ should have exponential order at least $-2 h$ when $s$ tends to minus infinity. This holds if one considers the first fundamental BVP for the strip, see Appendix B. However for the inverse problems considered here one has to convert the solution to a form suitable for the application of the inverse FT. For this purpose let us seek the displacements on $y=0$ as the following integral: 


$$
w(x)=\frac{1}{y} \int_{-\infty}^{\infty} \frac{\chi(t)}{1+\exp \left(\frac{\pi}{y}(t-x)\right)} d t, \quad-\infty<x<\infty, \quad 0<y \leq 2 h .
$$

Then bearing in mind the following integrals (e.g., Bateman \& Erdelyi, 1954)

$$
\int_{-\infty}^{\infty} \frac{e^{-i s t}}{1 \pm e^{-t}} d t=\frac{-i \pi}{\sinh (\pi s)}\left\{\begin{array}{c}
1 \\
\cosh (\pi s)
\end{array}, \quad-1<\operatorname{Im} s<0\right.
$$

by the convolution theorem one finds:

$$
\hat{w}(s) \sinh (y s)=-i \hat{\chi}(s), \quad-1<\operatorname{Im} s<0, \quad 0<y \leq 2 h .
$$

We now make use of (25), the convolution theorem and the second integral in (24) to evaluate the following integral:

$$
\begin{aligned}
W(x, y) & =\frac{1}{2 \pi} \int_{-\infty}^{\infty} \hat{w}(s) e^{-s y} e^{i s x} d s=\mathbf{F}^{-1}\left[\hat{w}(s) e^{-s y}\right]=\mathbf{F}^{-1}[\hat{w}(s)(\cosh (s y)-\sinh (s y))]= \\
& \left.\left.=\mathbf{F}^{-1}[\hat{w}(s) \sinh (s y)(\operatorname{coth}(s y)-1))\right]=-i \mathbf{F}^{-1}[\hat{\chi}(s)(\operatorname{coth}(s y)-1))\right]= \\
& -i \mathbf{F}^{-1}[\hat{\chi}(s) \operatorname{coth}(s y)]+i \chi(x)=\frac{1}{y} \int_{-\infty}^{\infty} \frac{\chi(t) d t}{1-\exp \left(\frac{\pi}{y}(t-x)\right)} d t+i \chi(x) .
\end{aligned}
$$

By introducing the substitutions $\eta=e^{-\frac{\pi}{y} t}, \xi=e^{-\frac{\pi}{y} x}$ and the new function $\chi_{0}(\eta)=\chi\left(-\frac{y}{\pi} \ln \eta\right)$ one can transform the latter integral to the form:

$$
\Lambda_{0}(\zeta)=\frac{1}{\pi} \int_{0}^{\infty} \frac{\chi_{0}(\eta)}{\eta-\xi} d \eta+i \chi_{0}(\xi)=2 i \Lambda_{0}^{+}(\xi), \quad 0<\xi<\infty .
$$

where $\Lambda^{+}(\xi)$ is the boundary value of the holomorphic function

$$
\Lambda_{0}(\zeta)=\frac{1}{2 \pi i} \int_{0}^{\infty} \frac{\chi_{0}(\eta)}{\eta-\zeta} d \eta
$$

Therefore, the solution for the complex potentials in (22) can be presented in the form:

$$
\left(\begin{array}{l}
\Phi(z) \\
\Omega(z)
\end{array}\right)=\frac{P}{(1+\kappa) z}\left(\begin{array}{c}
1 \\
\kappa-1
\end{array}\right)+\frac{2 i}{(1+\kappa)}\left(\begin{array}{c}
\Lambda(z) \\
-\Lambda(z)+\bar{\Lambda}(z)
\end{array}\right), \quad 0<\operatorname{Im} z \leq 2 h .
$$

Here $\Lambda(z)$ is another holomorphic function obtained by the change of variable in the function $\Lambda_{0}(\xi)$. The expressions above present an analytical solution of the problem in terms of the auxiliary function $\chi_{0}(\xi)$. This should be determined by inversion of the integral equation (23). By introducing a new function $w_{0}(\xi)=w\left(-\frac{y}{\pi} \ln \xi\right)$ one transforms (23) to the form of the Stieltjes integral:

$$
w_{0}(\xi)=\frac{1}{\pi} \int_{0}^{\infty} \frac{\chi_{0}(\eta)}{\eta+\xi} d \eta, \quad 0<\xi<\infty .
$$

Thus, the problem is reduced to the inversion of the Stieltjes transform, which according to Widder (1946), can be presented in the following complex form: 


$$
\chi_{0}(\xi)=\frac{1}{2 \pi i} \lim _{\rho \rightarrow 0}\left(w_{0}(-\xi-i \rho)-w_{0}(-\xi+i \rho)\right) .
$$

The formulas (28)-(31) complete the solution of the problem for the strip.

\subsection{Treatment of the surface displacements monitored at discrete points}

It should be noted that the representation (30) allows one to use displacement measurements in discrete form in order to determine the auxiliary function $\chi_{0}(\xi)$ entering into the solution. Although we have a formal inversion formula (31), it is not helpful for practical calculations because one needs to convert the discrete data into continuous data. This can be done by a number of ways. For instance, while obtaining solutions for the wedge-like domains Galybin (1999) has shown that the Laguerre polynomials can be used for fitting the data followed by the use of analytical solution similar to (31) to obtain the auxiliary function. This however does not fully eliminate instability in calculations as the Stieltjes transform belongs to a wide class of Fredholm integral equations of the first kind that, as is well known, is a classical example of the integral equations possessing nonstable solutions, Tikhovov \& Arsenin (1977). Another way is to find a numerical solution of (30) at certain nodes and to use interpolation in order to find smooth approximation for the auxiliary function $\chi_{0}(\xi)$.

It is understood that the displacement measurements cannot be performed over the semi-infinite interval and that the accuracy of the displacement measurements far from the point of concentrated load application is questionable. Therefore instead of (30) let us analyse the following integral equation reduced to a finite contour:

$$
w_{0}(\xi)=\frac{1}{\pi} \int_{0}^{L} \frac{\chi_{0}(\eta)}{\eta+\xi} d \eta, \quad 0<\xi<L .
$$

It is evident that (32) retains all the features of inverse problems with unstable solutions.

Let us seek the solution of (30) in the form of polynomial of $n$-th order with unknown coefficients $c_{k}(k=0, \ldots n)$ that provides necessary smoothness of the solution:

$$
\chi_{0}(\eta) \approx P_{n}\left(\frac{\eta}{L}\right)=c_{0}+c_{1} \frac{\eta}{L}+\ldots+c_{n}\left(\frac{\eta}{L}\right)^{n}, \quad 0<\frac{\eta}{L}<1 .
$$

Substitution of (33) into (32) results in

$$
w_{0}(L \gamma)=\sum_{k=0}^{n} c_{k} I_{k}(\gamma), \quad I_{k}(\gamma)=\frac{1}{\pi} \int_{0}^{1} \frac{\tau^{k}}{\tau+\gamma} d \tau, \quad 0<\gamma<1 .
$$

The following recurrent formulas can be readily derived:

$$
I_{0}(\gamma)=\frac{1}{\pi} \ln \frac{1+\gamma}{\gamma}, \quad I_{1}(\gamma)=\frac{1}{\pi}\left(1-\gamma \ln \frac{1+\gamma}{\gamma}\right), \quad I_{k}(\gamma)=\frac{1}{\pi} \frac{k \gamma+k-1}{k(k+1)}-\gamma^{2} I_{k-2}(\gamma), \quad k=2 \ldots n .
$$

We use (35) to analyse stability of recovering the first four trail functions $\tau^{k}$ from the solution of the integral equation:

$$
\frac{1}{\pi} \int_{0}^{1} \frac{\chi_{0}(\tau)}{\tau+\gamma} d \tau=I_{k}(\gamma)(1+\varepsilon \lambda(\gamma)), \quad 0<\gamma<1, \quad k=0 \ldots 3,
$$

for which the right hand side is subjected to errors $\lambda(y)$ randomly distributed on $(-1,1), \varepsilon$ is the level of noise.

Let us apply (34) to the left hand side of (36) and introduce a set of collocation points $\gamma_{m}$ equidistant 
on $(0,1)$. Then (36) reduces to the following system of linear algebraic equations for the determination of the unknown coefficients $c_{k}$ :

$$
\sum_{j=0}^{n} c_{j} I_{j}\left(\gamma_{m}\right)=W_{k}^{m}, \quad W_{k}^{m}=I_{k}\left(\gamma_{m}\right)\left(1+\varepsilon \lambda_{m}\right), \quad \gamma_{m}=\frac{m}{M+1}, \quad m=1 \ldots M, \quad k=0 \ldots 3 .
$$

In all calculations below $\lambda_{m}$ are Gaussian errors and $\varepsilon=0.05, n=4, M=40$.

The calculations have shown that the solution of (37) is highly sensitive to errors. Therefore the SVD regularisation in the form as discussed in detail in Galybin (2008) has been applied. The results of recovering the trial function are shown in Fig. 3 by dashed lines, for visual comparison the "ideal" functions $\gamma^{k}\left({ }_{\mathrm{k}}=0 \ldots 3\right)$ are shown by solid lines.
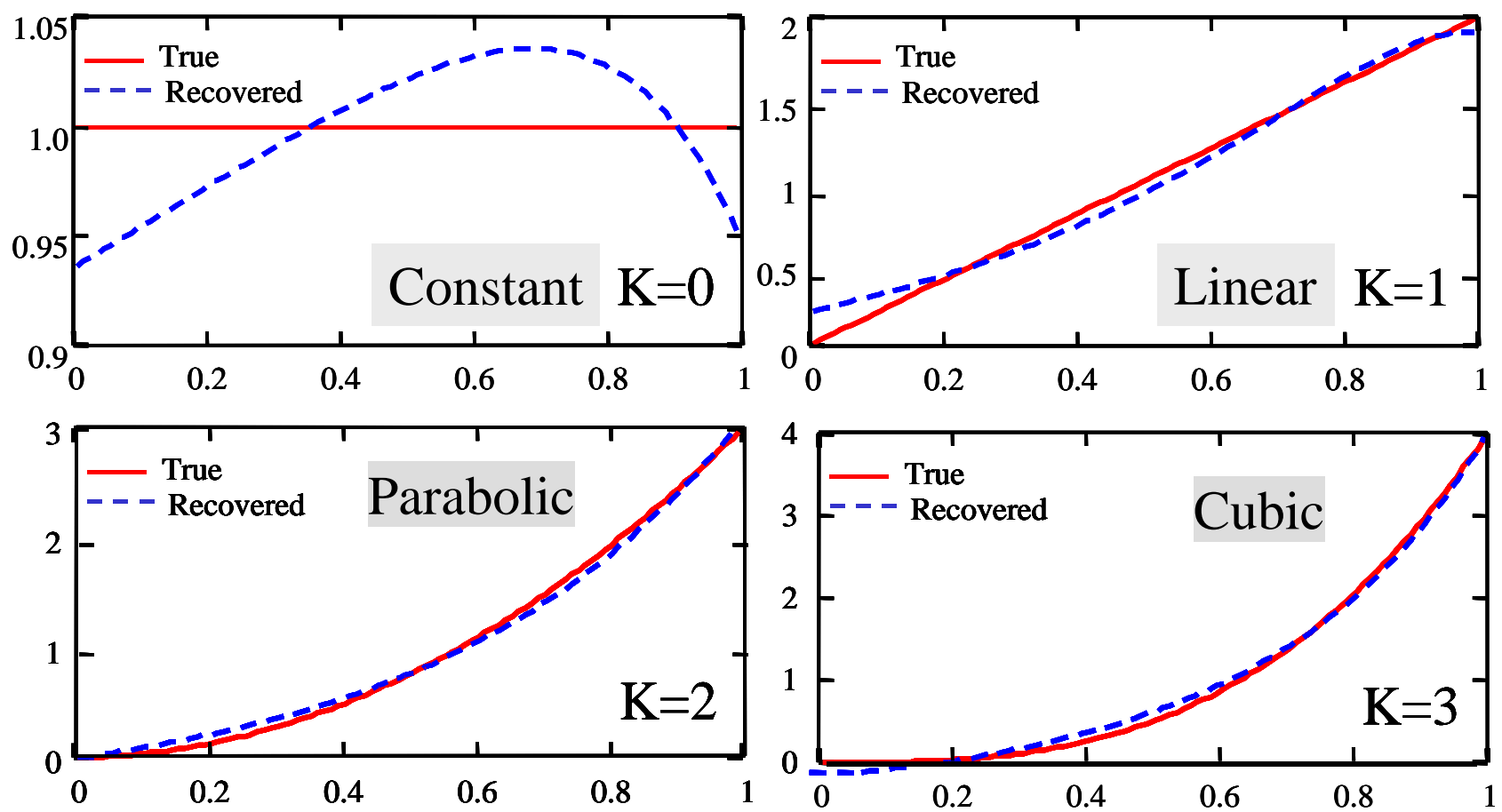

Figure 3 The results of recovering the trail functions $\gamma^{k}(k=0,1,2,3)$ shown by dashed lines, the ideal ones are shown by solid lines.

\section{Conclusions}

This paper presents the solution of a Cauchy-type problem for plane elasticity in respect of a strip. In contrast to standard formulations, the boundary conditions (stresses) are unknown on one side of the strip, with the displacements given on the other side, which is a stress-free boundary apart from the point of application of the concentrated load. It is shown that this problem has a unique solution. The solution is found by application of the Fourier transform with respect to the variable directed along the strip boundaries. In contrast to the standard approach, the Fourier transform is applied to the Kolosov-Muskhelishvili formulas in order to find two holomorphic functions. They are obtained in analytical form by applying the inverse Fourier transform that also requires the inversion of the Stieltjes integral. This presents a numerically unstable operation. It has been shown that the SVD regularisation is capable of providing stable solutions for the inversion of the Stieltjes integral. The results of this study can be used for detection of delamination on the interface between a coating and a substrate, which is important for providing non-destructive control of integrity of coated structures.

Acknowledgements: This work was completed without any external funding, although some internal funding from Keele University supported a visit to Keele by AG. 


\section{APPENDIX A. Application of the Fourier transform to the system of integral equations.}

Let us show how equations (21) can be directly derived from the system (9) for a strip with the boundaries $\Gamma_{1}=\{z=t:-\infty<t<\infty\}, \quad \Gamma_{2}=\{z=t+2 i h:-\infty<t<\infty\}$. The system of equations (9) takes the form:

$$
\begin{aligned}
& \varphi_{1}(t)+\frac{1}{\pi i} \int_{-\infty}^{\infty} \frac{\varphi_{1}(t)}{x-t} d t=\frac{1}{\pi i} \int_{-\infty}^{\infty} \frac{\varphi_{2}(t)}{x-t-2 i h} d t, \quad \text { on } \quad \Gamma_{1}, \quad|x|<\infty . \\
& \frac{-1}{\pi i} \int_{-\infty}^{\infty} \frac{\varphi_{1}(t)}{x-t+2 i h} d t=\varphi_{2}(x)-\frac{1}{\pi i} \int_{-\infty}^{\infty} \frac{\varphi_{2}(t)}{x-t} d t, \quad \text { on } \quad \Gamma_{2}, \quad|x|<\infty .
\end{aligned}
$$

Here the opposite signs of the integrals on the right hand sides of both equations are due to reversing the direction of integration over $\Gamma_{2}$.

The kernels in the integrals in (A1) and (A2) are of the convolution type. Taking into account that

$$
\mathbf{F}\left(\frac{1}{x}\right)=-i \pi \operatorname{sgn}(s), \quad \mathbf{F}\left(\frac{1}{x+2 i h}\right)=-2 i \pi e^{-2 h s}\left\{\begin{array}{ll}
1, & s>0 \\
0, & s<0
\end{array}, \quad \mathbf{F}\left(\frac{1}{x-2 i h}\right)=-2 i \pi e^{2 h s} \begin{cases}0, & s>0 \\
1, & s<0\end{cases}\right.
$$

one obtains the following system of equations after application of FT to (A1) and (A2):

$$
(1-\operatorname{sgn}(s)) \hat{\varphi}_{1}(s)=-2 e^{2 h s} \hat{\varphi}_{2}(s)\left\{\begin{array}{ll}
0, & s>0 \\
1, & s<0
\end{array}, \quad(1+\operatorname{sgn}(s)) \hat{\varphi}_{2}(s)=2 e^{-2 h s} \hat{\varphi}_{1}(s)\left\{\begin{array}{ll}
1, & s>0 \\
0, & s<0
\end{array}\right. \text {. }\right.
$$

It is evident that (A4) is equivalent to $\hat{\varphi}_{1}(s)=e^{2 h s} \hat{\varphi}_{2}(s)$, Q.E.D.

\section{APPENDIX B. First fundamental BVP for the strip}

Let us consider an elastic strip $\mathrm{X}=\{z=x+i y:-\infty<x<\infty, 0 \leq y \leq 2 h\}$ with the boundaries $\Gamma_{1}=\{z=t:-\infty<t<\infty\}, \quad \Gamma_{2}=\{z=t+2 i h:-\infty<t<\infty\}$. The strip is loaded with normal and shear stresses $f_{1}(x)=q_{1 y}(x)+i q_{1 x}(x)$ on $\Gamma_{1}$ and stresses $f_{2}(x)=q_{2 y}(x)+i q_{2 x}(x)$ on $\Gamma_{2}$.

Let us apply FT (14) to the stress vector and use the properties in (15)-(16). Then on $y=0$ :

$$
\mathbf{F}\left(\sigma_{y}+i \tau_{x y}\right)=\overline{\hat{\Phi}(-s)}+\hat{\Phi}(s)+\hat{\Omega}(s)=f_{1}(s),
$$

while on $\mathrm{y}=2 h$ :

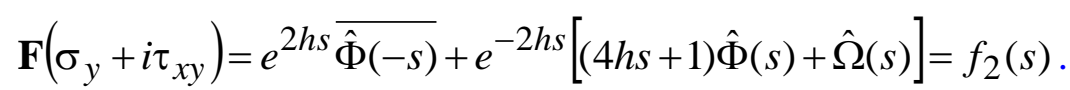

Exclude $\hat{\Omega}(s)$ from the system (B1)-(B2):

$$
\begin{gathered}
\hat{\Omega}(s)=f_{1}(s)-\overline{\hat{\Phi}(-s)}-\hat{\Phi}(s), \\
{\left[e^{2 h s}-e^{-2 h s} \sqrt{\hat{\Phi}(-s)}+4 h s e^{-2 h s} \hat{\Phi}(s)=f_{2}(s)-e^{-2 h s} f_{1}(s) .\right.}
\end{gathered}
$$

Complex conjugation of (B4) followed by the replacement the variable $s$ by $-s$ leads to the following system for the determination of $\hat{\Phi}(s)$ : 


$$
\begin{aligned}
& 4 h s e^{-2 h s} \hat{\Phi}(s)+\left\lfloor e^{2 h s}-e^{-2 h s} \overline{\hat{\Phi}(-s)}=f_{2}(s)-e^{-2 h s} f_{1}(s)\right. \\
& {\left[e^{-2 h s}-e^{2 h s}\right] \hat{\Phi}(s)-4 h s e^{2 h s} \overline{\hat{\Phi}(-s)}=\overline{f_{2}(-s)}-e^{2 h s} \frac{f_{1}(-s)}{}}
\end{aligned}
$$

From (B5) it follows that

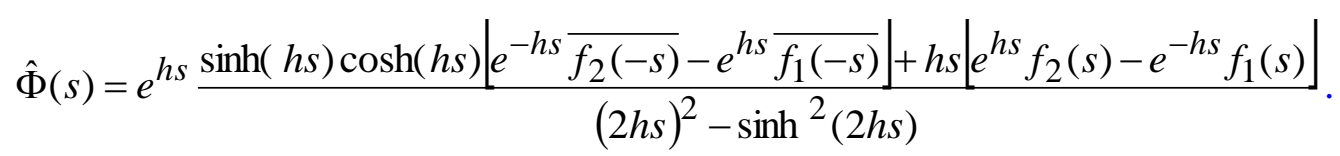

Therefore for $0 \leq y \leq 2 h$ :

$$
\mathbf{F}(\Phi(z))=e^{(h-y) s} \frac{\sinh (h s) \cosh (h s)\left\lfloor e^{-h s} \overline{f_{2}(-s)}-e^{h s} \overline{f_{1}(-s)}\right\rfloor+h s\left\lfloor e^{h s} f_{2}(s)-e^{-h s} f_{1}(s)\right\rfloor}{(2 h s)^{2}-\sinh ^{2}(2 h s)} .
$$

The FT of the potential $\Omega(\mathrm{z})$ is found by substitution of (B6) into (B3) followed by multiplication by $\exp (-y s)$. Therefore the solution of the BVP in terms of the FT is complete. It is evident from the solution that both $\mathbf{F}(\Phi(\mathrm{z}))$ and $\mathbf{F}(\Omega(\mathrm{z}))$ remain bounded when $s \rightarrow \pm \infty$, which provides the existence of solution (we assume that the FT of the loads are bounded as well due to physical restrictions). The functions $\mathbf{F}(\Phi(\mathrm{z}))$ and $\mathbf{F}(\Omega(\mathrm{z}))$ have poles at the pole where the denominator in (B6) vanishes formally, in particular at the origin, $s=0$. This indicates that the displacements and their contour derivatives can formally be unbounded when $x \rightarrow \pm \infty$. This imposes further restrictions on the applied loads to the displacement derivatives bounded.

The solution for the displacement vector in terms of the stresses follows from (B6) and the FT of (7) from the main text. It can be presented as follows:

$$
\begin{aligned}
& \mathbf{F}\left[2 G\left(u^{\prime}+i v^{\prime}\right)\right]=-\overline{f_{1}(-s)}+ \\
& \quad+(\kappa+1) e^{h s} \frac{\sinh (h s) \cosh (h s)\left[e^{-h s} \overline{f_{2}(-s)}-e^{h s} \overline{f_{1}(-s)}\right]+h s\left[e^{h s} f_{2}(s)-e^{-h s} f_{1}(s)\right]}{(2 h s)^{2}-\sinh ^{2}(2 h s)}, \quad y=0 . \\
& \mathbf{F}\left[2 G\left(u^{\prime}+i v^{\prime}\right)\right]=-\overline{f_{2}(-s)}+ \\
& \quad+(\kappa+1) e^{-h s} \frac{\sinh (h s) \cosh (h s)\left[e^{-h s} \overline{f_{2}(-s)}-e^{h s} \overline{f_{1}(-s)}\right]+h s\left[e^{h s} f_{2}(s)-e^{-h s} f_{1}(s)\right]}{(2 h s)^{2}-\sinh ^{2}(2 h s)}, \quad y=2 h .
\end{aligned}
$$

Formula (B8) or (B9) can serve as "ideal solutions" for generating synthetic data on displacement monitoring on the stress free surface.

Let $f_{2}=0$ and $2 h=1$, then on $y=0$ :

$$
\mathbf{F}\left[2 G\left(u^{\prime}+i v^{\prime}\right)\right]=-\overline{f_{0}(-s)}-\frac{\kappa+1}{2} \frac{e^{s} \sinh (s) \overline{f_{0}(-s)}+s f_{0}(s)}{s^{2}-\sinh ^{2}(s)} .
$$

For plane stress $(1+\kappa) / 2=2 /(1+v)$, by setting $s=-i p$ (where $p$ is a complex parameter) and $2 G=E /(1+v)(E$ is Young's modulus $)$, one finds:

$$
\frac{E}{2} \mathbf{F}\left[\left(u^{\prime}+i v^{\prime}\right)\right]=\frac{1-v}{2}\left(\hat{q}_{1 y}-i \hat{q}_{1 x}\right)+\frac{\left.\left(p^{2}+i \cos p \sin p\right)\right)\left(\hat{q}_{1 y}-i \hat{q}_{1 x}\right)+i p\left(\hat{q}_{1 y}+i \hat{q}_{1 x}\right)}{\sinh ^{2}(p)-p^{2}} .
$$

This special case fully coincides with the problem for the strip considered by Khrapkov (2001), who used the bilateral Laplace transform (it is reduced to FT by the substitution $s=-i p$ ).

Consider the case of a pair of concentrated normal loads of intensity $P_{0}$ that are applied co-axially at the points $\mathrm{z}=0$ and $\mathrm{z}=2 i h$ of the boundary, i.e. $f_{1}(\mathrm{~s})=f_{2}(\mathrm{~s})=P_{0}=\mathrm{const}\left(\operatorname{Im} P_{0}=0\right)$. Then it follows from (B8) and (B9) that 


$$
\mathbf{F}\left[2 G\left(u^{\prime}+i v^{\prime}\right)\right]=-P_{0}-2(\kappa+1) e^{h s} \sinh (h s) \frac{\sinh (h s) \cosh (h s)-h s}{(2 h s)^{2}-\sinh ^{2}(2 h s)} P_{0}, \quad y=0, \quad y=2 h .
$$

It is evident from (B12) that $s=0$ is not a pole of $\mathbf{F}\left[2 G\left(u^{\prime}+i v^{\prime}\right)\right] \rightarrow-\left(1+\frac{3}{16}(\kappa+1)\right) P_{0}, \quad s \rightarrow 0$, which justifies the statement that the contour derivatives of the displacements at infinity may vanish.

\section{References}

Bateman, H., Erdelyi, A. (1954). Tables of integral transforms, McGraw-Hill, New York.

Bonnet, M. Constantinescu, A. (2005). Inverse problems in elasticity. Inverse Problems, v.21, pp. R1-R50.

Bui, H. D. (1994). Inverse problems in the mechanics of materials: an introduction. Boca Raton, FL: CRC Press.

Erdogan F., Gupta, G. (1971). The stress analysis of multi-layered composites with a flaw. Jnt J Solids and Structures, v. 7 (8), pp.39-61.

Gakhov, F.D. (1990). Boundary value problems, Dover Publ., New York.

Galybin, A.N. (1999). A non-classical plane elastic boundary value problem. In Sarler et al (Eds). Moving Boundaries V. Computational Modelling of Free and Moving Boundary Problems (pp. 5968). WIT Press, Southampton, UK.

Galybin, A.N., (2001). A Method for determination of stress distributions in the process zone ahead of a 2D crack. In B. Sarler and C. A. Brebbia (Eds.), Moving Boundaries VI Computational Modelling of Free and Moving Boundary Problems (pp. 243-252). WIT Press, Southampton, UK.

Galybin, A.N. (2008). An inverse problem of elastostatics in mechanics of composites. Composite Science and Technology, v.68(5), pp.1188-1197.doi:10.1016/j.compscitech.2007.05.027.

Galybin, A.N. (2011). Boundary value problems of plane elasticity involving orientations of displacements and tractions. Journal of Elasticity, v.102: pp. 15-30.doi:10.1007/s10659-010-9259-4

Galybin, A. N. (2016). Contact inverse problem for an elastic half-space. Engineering Analysis with Boundary Elements, v. 68, pp. 35-41. doi: 10.1016/j.enganabound.2016.03.019

Galybin, A.N., Mukhamediev, Sh.A. (1999). Plane elastic boundary value problem posed on orientation of principal stresses. Journal of the Mechanics and Physics of Solids, v.47 (11): pp. 2381-2409. doi:10.1016/S0022-5096(99)00032-0

Gao, Z., Mura, T. (1991). Elasticity problems with partially overspecified boundary conditions. Int Journal of Engineering Science, v. 29 (6), pp. 685-692.

Hsieh, S.C., Mura, T. (1993). Nondestructive cavity identification in structures. J. Solids Structures, v.30 (12), pp.1579-1587.

Johnson, K.L. (1985). Contact mechanics. Cambridge University Press.

Kaplunov, J., Prikazchikov, D., Sultanova, L. (2018). Justification and refinement of Winkler-Fuss hypothesis. Z. Angew. Math. Phys., v, 69:80, 15p. doi.org /10.1007/s00033-018-0974-1

Khrapkov, A.A. (2001). Wiener-Hopf method in mixed elasticity theory problems. VNIIG, SanktPeterburg.

Kubo, S. (1988). Inverse problems related to the mechanics and fracture of solids and structures. JSME Int. J. 31, 157-166.

Maniatty, A. Zabaras, N. (1989). Method for solving inverse elasto-viscoplastic problems. Journal of Engineering Mechanics, v.115 (10), pp. 2216-2231.

Maniatty, A., Zabaras, N., Stelson, K. (1989). Finite element analysis of some elasticity problems. 
J. Engng. Mech. Division ASCE, v. 115, pp. 1302-1316.

Marin, L., L. Elliott, D.B. Ingham, Lesnic, D. (2001). Boundary element method for the Cauchy problem in linear elasticity. Engineering Analysis with Boundary Elements v. 25 (9), pp. 783-793.

Marin, L., Lesnic, D. (2002). Boundary element solution for the Cauchy problem in linear elasticity using singular value decomposition. Computer Methods in Applied Mechanics and Engineering. v.191 (6), pp. 3257-3270.

Marin, L., Lesnic, D. (2005). The method of fundamental solutions for inverse boundary value problems associated with the two-dimensional biharmonic equation. Mathematical and Computer Modelling, v. 42 (3-4), pp. 261-278.

Murakami, Y. (1987). Stress intensity factors handbook. Pergamon Press.

Muskhelishvili, N.I. (1963). Some basic problems of the mathematical theory of elasticity. P. Noordhoff, Groningen, the Netherlands.

Schnur, D., Zabaras, N.. (1990). Finite element solution of two-dimensional elastic problems using spatial smoothing. Int. J. Numer. Meth. Engng. v.30, pp.57-75.

Schwab, A. A. (1994). The inverse problem of elasticity theory: Application of the boundary integral equation for the holomorphic vector. Physics of the Solid Earth, v.30 (4), 342-348.

Shevljakov Yu.A. (1977). Matrix algorithms in the theory of elasticity of inhomogeneous media. Vischa Schola, Kiev-Odessa, 216 p. (in Russian)

Shvab, A.A. (1989). Incorrectly posed static problems of elasticity. Solid Mechanics, v.24, pp. 98106.

Tanaka, M., Masuda, Y. (1986). Boundary element method applied to some inverse problems. Engineering Analysis, v.3 (3), pp.138-143.

Tikhonov, A.N. \& Arsenin, V.Y. (1977). Solution of Ill-Posed Problems, New York: Winston, Wiley

Tsvelodub, I. Yu. (2000). An inverse problem for an elastic medium containing a physically nonlinear inclusion. J. Appl. Maths Mechs., v.64 (3), pp. 407-412.

Widder, V.D. (1946). The Laplace transform, Princeton University Press, Princeton.

Yeih, W.C., Koya, T., Mura, T. (1993). An inverse problem in elasticity with partially overspecified boundary conditions. I. Theoretical approach. Trans. ASME J. Appl. Mech. v.60, pp. 595-600.

Zabaras, N., Morellas, V., Schnur, D. (1989). Spatially regularized solution of inverse elasticity problems using the BEM. Comm. Appl. Numer. Meth. v.5, pp.547-553. 